\title{
CONSECUENCIAS DEL RDL 20/2012 \\ EN MATERIA DE DESEMPLEO: ESTUDIO E IMPACTO JURÍDICO-ECONÓMICO Y SOCIAL SOBRE EL SUBSIDIO DE 52 AÑOS AL ACTUAL DE 55 AÑOS
}

\author{
Miguel Jaime Cano Esquibel \\ Doctorando en Derecho-Dpto. de Derecho del Trabajo y de la Seguridad Social. \\ Universidad de Alicante-UA \\ Colaborador Honorífico del Departamento de Ciencia Jurídica \\ de la Universidad Miguel Hernández de Elche-UMH
}

DOI: $10.1387 /$ lan-harremanak.15423

\section{ABSTRACT}

Con la publicación en el Boletín Oficial del Estado número 168 el 14 de julio de 2012 del Real Decreto Ley 20/2012 de 13 de julio entre otras muchas y diversas materias el que antes se llamaba "subsidio para mayores de 52 años" se incrementaba la edad de los 52 a los 55 años para los trabajadores que no tengan responsabilidades familiares, hayan cotizado al menos 6 años y acrediten los requisitos, excepto el de la edad, para el acceso a cualquier otro tipo de prestación contributiva de jubilación del sistema.

En segundo lugar, se modifica el subsidio por desempleo, llamado de "prejubilación", la norma establece que a la edad de 55 años ha de haberse cumplido en la fecha del agotamiento de la prestación por desempleo o del subsidio por desempleo; o tener 
cumplida esa edad en el momento de reunir los requisitos para acceder a cualquier otro subsidio de desempleo o cumplirla durante su percepción.

En cualquier caso, para este subsidio se pone un nuevo término: el momento en que el trabajador alcance la edad que le permita acceder a la pensión contributiva de jubilación en cualquiera de sus modalidades. Con ello se vuelve a la redacción original del artículo 216.3 LGSS, cuando el subsidio se extendia hasta que el trabajador alcanzara la edad que le permitiera acceder a la pensión contributiva de jubilación en cualquiera de sus modalidades, con la aplicación de los correspondientes coeficientes reductores, en su caso.

Ha de recordarse que esta nueva regla desde el día 16 de julio de 2012 esta haciendo mucho daño a los trabajadores que acceden a la nueva prestación ya que han de tenerse en cuenta las nuevas reglas de integración de lagunas, las de determinación de cuantía a minimos (limitada a la cuantía de las prestaciones no contributivas), ambas establecidas por la Ley 27/2011 de reforma del sistema de Seguridad Social, y por supuesto, la regla de que la pensión minima para los menores de 65 años es inferior a la común establecida.

Además, es importante por cuanto lleva consigo una reducción real de las futuras pensiones de jubilación de los que sean beneficiarios de subsidios por desempleo mayores de 55 años, la base de cotización por la que cotizaba el SPEE-SEPE era el $125 \%$ de la base minima de cotización. Con la reforma, todos los subsidios por desempleo que coticen por la contingencia de jubilación lo harán por el $100 \%$. El ahorro para el sistema público es, pues doble: de un lado el SPEE ahorra en cotizaciones con la reforma normativa y de otro lado, en el futuro el sistema de seguridad social ahorrará en prestaciones publicas.

Palabras clave: Subsidio, igualdad, justicia social, prestaciones publicas, crisis económica.

With the publication in the B.O.E. (Official State Gazette) number 168 on July 14, 2012 Real Decree Law 20/2012 of 13 July among many and various subjects which used to be called "Allowance for over 52 years, " the age of 52 was increased to 55 years for workers who have family responsibilities, have contributed at least 6 years evidencing the requirements except age for access to any other provision contributory retirement system.

Second, unemployment benefits, called "early retirement" is changed, the standard It states that at the age of 55 years must be met at the date of expiry of the unemployment benefits or unemployment benefits; or have fulfilled that age at the time to qualify for access to any unemployment or fulfill during their perception. 
In any case, for this grant gets a new term: the time the worker reaches the age where you have access to the contributory retirement pension in any modalities. This is back to the original wording of Article 216.3 LGSS, when the subsidy extended until the worker reached the age to be allowed access to the pension contributory retirement in all its forms, with the implementation of the corresponding reduction factors, if any.

It must be remembered that this new rule from the day July 16, 2012 is doing great harm to workers who have access to the new provision must be considered as the new rules integration gaps, determining a minimum amount (limited to the amount of non-contributory benefits), both established by Law 27/2011 of reform Social Security, and of course, the rule that the minimum pension for those under 65 is below the common set.

It is also important because it brings a real reduction of future pension retirement who are beneficiaries of unemployment benefits over 55 years, the base Quote why the SPEE-SEPE traded was $125 \%$ of the minimum contribution base. With the reform, all unemployment benefits listed for the contingency of retirement will do for $100 \%$. The savings to the public system is thus twofold: on one side the SPEE saves $Q$ uotes regulatory reform and on the other hand, in the future the social security system save on public services.

Key Words: Subsidy, equality, social justice, public benefits, economic crisis. 


\section{Introducción}

El término desempleo alude a la falta de trabajo. Una persona desempleada es un sujeto en edad de trabajar, es decir, que forma parte de la población activa y que no consigue trabajo a pesar de buscarlo de manera activa y continua.

Tal y como está previsto en el Título I, Capítulo II, Artículo 35 de la Constitución Española: «1. Todos los españoles tienen el deber de trabajar y el derecho al trabajo, a la libre elección de profesión u oficio, a la promoción a través del trabajo y a una remuneración suficiente para satisfacer sus necesidades y las de su familia, sin que en ningún caso pueda hacerse discriminación por razón de sexo.»

Por tanto, según esta definición se puede decir que el desempleo es la imposibilidad de trabajar pese a la voluntad de la persona. Así pues, la población realmente activa, es la que está ocupada o buscando empleo.

Se puede clasificar el desempleo en cuatro clases:

- Desempleo Cíclico: es aquel que aparece en épocas de crisis económicas o de recesión y por lo general no es demasiado extenso en el tiempo.

- Desempleo Estacional: aparece por la fluctuación estacional de la oferta y la demanda, dependiendo de las estaciones del ańo.

- Desempleo Friccional: surge por el desacuerdo entre empleado y empleador. Es decir, las condiciones del puesto de trabajo no satisfacen al empleado por tanto abandona su empleo.

- Desempleo Estructural: es considerado como el más grave. Se trata de un desajuste entre la oferta y la demanda de trabajo. Es decir, el número de puestos de trabajo disponibles en un sistema económico es menor que el número de personas que buscan empleo.

Dentro de la política de recortes que lleva imponiendo el actual Gobierno, las prestaciones por desempleo no han salido indemnes. Muy al contrario, tanto en el nivel contributivo como en el nivel asistencial se han aplicado rebajas importantes. 
En algunos casos con tal intensidad, que cuestionan el sistema de protección por desempleo existente para determinados colectivos en nuestro país y reduce el artículo 41 de nuestra Constitución que establece la garantía de prestaciones suficientes ante situaciones de necesidad, especialmente, en caso de desempleo; a una desgraciada ironía.

Un caso paradigmático es el que corresponde al Subsidio por desempleo para mayores de 52 años — ahora de 55-. Este subsidio ha sido colocado en el punto de mira por el Consejo de Ministros, prueba de ello es que tanto el Real Decreto-ley 20/2012, del 13 de julio, como el más reciente Real Decretoley 5/2013, de 15 de marzo, han recogido modificaciones sobre el mismo que vienen a desmantelarlo y a dejar sin cobertura de la acción protectora a un colectivo de desempleados y parados de larga duración con muchas carencias y problemas en su ultimos años de vida laboral.

\section{El subsidio de desempleo y su acción protectora. Antes y después del RDL 20/2012}

No debemos olvidar que este subsidio buscaba, y supuestamente aún busca, cubrir una situación de desempleo, una vez agotada la prestación contributiva, de personas que cuentan con una edad que dificulta severamente la inserción en el mercado laboral y, por lo tanto, donde la situación de necesidad de cobertura se pone de manifiesto sin ninguna duda. Por otro lado, se trata de un subsidio ligado a un largo período de cotización desde el momento en que entre los requisitos para su acceso se encuentra el tener período suficiente cotizado para acceder a la jubilación, lo que impide, en buena medida, mecánicas perversas en su utilización y no es un segmento de población en el que quepa encontrar bolsas significativas de fraude alguno.

¿El porqué de este afán del Gobierno en devaluar un subsidio que deberíamos considerar una de las columnas fundamentales en las que se soporta el nivel asistencial de protección por desempleo? Es difícil de entender, salvo que pretendamos poner en valor los hipócritas argumentos que se esgrimen en la norma utilizada para efectuar la escabechina: incentivas el alargamiento de la vida laboral. Se les olvidó indicar que el incentivo era a palos.

Para conocer la dimensión del recorte efectuado, vamos a recoger, de una forma que permita el trabajo de comparación, los requisitos de acceso a este subsidio antes de julio de 2012 y en la actualidad, momento en el que se suman las modificaciones efectuadas por los dos reales decreto-ley mencionados. 


\begin{tabular}{|c|c|c|c|}
\hline & Factor & Antes & Ahora \\
\hline 1 & $\begin{array}{c}\text { Edad } \\
\text { (art. 17.siete } \\
\text { RDL 20/2012) }\end{array}$ & 52 años. & 55 años. \\
\hline 2 & $\begin{array}{l}\text { Duración } \\
\text { (art. 17.ocho } \\
\text { RDL 20/2012) }\end{array}$ & $\begin{array}{l}\text { A los } 65 \text { años. En todo caso, la } \\
\text { decisión la gobernaba el traba- } \\
\text { jador o trabajadora. }\end{array}$ & $\begin{array}{l}\text { Cuando se pueda acceder a } \\
\text { cualquier tipo de jubilación. } \\
\text { También la anticipada en cual- } \\
\text { quiera de sus modalidades. }\end{array}$ \\
\hline 3 & $\begin{array}{l}\text { Cotización por } \\
\text { jubilación } \\
\text { (art. 17.diez.3 } \\
\text { RDL 20/2012) }\end{array}$ & $\begin{array}{l}\text { Cotizaba por el } 125 \% \text { de la } \\
\text { base mínima vigente. }\end{array}$ & $\begin{array}{l}\text { Cotiza por la base mínima vi- } \\
\text { gente. }\end{array}$ \\
\hline 4 & $\begin{array}{l}\text { Forma de acceso } \\
\quad(\text { art. } 17 . \text { siete } \\
\text { RDL 20/2012) }\end{array}$ & $\begin{array}{l}\text { Cumpliendo la edad y estando } \\
\text { inscrito. Lógicamente, hay que } \\
\text { cumplir los requisitos de ac- } \\
\text { ceso a jubilación y rentas. }\end{array}$ & $\begin{array}{l}\text { La edad debe cumplirse per- } \\
\text { cibiendo algún tipo de presta- } \\
\text { ción por desempleo. No sirve } \\
\text { la mera permanencia en la ins- } \\
\text { cripción por desempleo. }\end{array}$ \\
\hline 5 & $\begin{array}{c}\text { Trabajo a tiempo } \\
\text { parcial } \\
\text { (art. 17.nueve } \\
\text { RDL 20/2012) }\end{array}$ & $\begin{array}{l}\text { Se percibía el subsidio al } \\
100 \% \text {. }\end{array}$ & $\begin{array}{l}\text { Se percibe el subsidio con el } \\
\text { porcentaje del último contrato } \\
\text { a tiempo parcial. }\end{array}$ \\
\hline 6 & $\begin{array}{c}\text { Rentas y patrimonio } \\
\text { (art. 17.siete } \\
\text { RDL 20/2012 }\end{array}$ & $\begin{array}{l}\text { Al patrimonio que no produ- } \\
\text { cía rentas se le aplicaba el } 50 \% \\
\text { de tipo de interés legal del di- } \\
\text { nero a la hora de determinar } \\
\text { las rentas del beneficiario. }\end{array}$ & $\begin{array}{l}\mathrm{Al} \text { patrimonio que no produce } \\
\text { rentas se le aplica el } 100 \% \text { del } \\
\text { tipo de interés legal. }\end{array}$ \\
\hline 7 & $\begin{array}{l}\text { Unidad familiar } \\
\text { (Disp. Final 1.a.uno } \\
\text { RDL 5/2013) }\end{array}$ & $\begin{array}{l}\text { No se tienen en cuenta los in- } \\
\text { gresos de la unidad familiar. }\end{array}$ & $\begin{array}{l}\text { Los ingresos de la unidad fa- } \\
\text { miliar, si superan, divididos } \\
\text { por el número de miembros, el } \\
75 \% \text { del SMI provocan la de- } \\
\text { negación del subsidio. }\end{array}$ \\
\hline
\end{tabular}

En definitiva:

- Se ha retrasado en tres años el acceso a dicho subsidio dejando abandonados a los desempleados y desempleadas que se encuentran en dicha franja de edad.

- Pero no solo eso, además ahora, para acceder al subsidio debo cumplir los años percibiendo cualquier otro tipo de prestación. No sirve con cumplir los 55 años y estar en desempleo, en dicho caso el trabajador o 
trabajadora deberá encontrar otro empleo, cotizar el período suficiente para acceder a cualquier tipo de prestación y cuando finalice, sólo en ese caso, podrá acceder al subsidio de mayor de 55 años.

- En lo que afecta a la fecha de finalización, se ha encogido sensiblemente el período ele cobertura al quedar ligado a la fecha en la que la persona beneficiaria puede acceder a cualquier tipo de jubilación. Con ello estamos descapitalizando al/la trabajador/a que se en la obligación de asumir una jubilación con importantes coeficientes reductores o a quedar sin cobertura alguna por desempleo desde dicha fecha.

- Se reduce en un $25 \%$ la cotización, se devalúa la pensión que será posible obtener a futuro y se encarece de forma nada despreciable la posibilidad de mantener las bases reguladoras a través de un convenio especial con la Seguridad Social.

- Se penaliza a quienes han trabajado a tiempo parcial, quienes está situados en una posición mas débil en el sistema, percibirán el subsidio en función del porcentaje trabajado. Es más, se desincentiva la colocación cuando ésta sea a tiempo parcial de quienes perciban este subsidio en la actualidad, ya que si ahora la cobran al $100 \%$, la generación de un nuevo desempleo derivado de una contratación a tiempo parcial provocaría que el subsidio de mayor de 55 ańos que debieran solicitar posteriormente, se percibiese reducido en función del contrato a tiempo parcial.

- Se endurecen los criterios de renta, aplicando el $100 \%$ del tipo de interés legal sobre cualquier tipo de patrimonio no productivo.

- Se da una nueva vuelta de tuerca y se penaliza a quienes pertenecen a una unidad familiar, contabilizando los ingresos de la misma, cuando hasta la fecha solo se tenían en cuenta los ingresos del propio beneficiario/a. Una medida irracional que parece querer propiciar la separación matrimonial como fórmula para poder acceder a este subsidio.

Como puede apreciarse, se ha debilitado hasta el extremo la protección de los trabajadores y trabajadoras de mayor edad que arrastren una situación de desempleo que se alargue en el tiempo. Un colectivo al que se le castiga doblemente endureciendo hoy las condiciones para poder acceder a una prestación por desempleo y estableciendo toda una verdadera ingeniería administrativa destinada a rebajar las expectativas económicas de la pensión de jubilación que pudiese corresponderle en un futuro.

Un castigo que recae con más fuerza sobre los mas débiles, sobre quienes tienen peores perspectivas de colocación, aumentado la desigualdad y profundizando las situaciones de pobreza. 


\section{Particularidades y comentarios de la reforma del subsidio de "prejubilación» de los 52 a 55 años, desprotección del sistema en el futuro}

En esta ocasión, es el Real Decreto Ley 20/2012 el que concentra los últimos cambios habidos fundándose en que «las continuas modificaciones que ha sufrido el sistema le han restado coherencia, desincentivando en ocasiones la vida activa y generando situaciones contrarias al principio de equidad» (preámbulo).

Con ello «desvirtúa la finalidad del sistema en su conjunto y pone en riesgo la protección de los más necesitados, que se ven afectados por el impacto del diseño actual en la sostenibilidad del sistema de protección social». Por tanto - concluye-, «se introducen una serie de medidas que tratan de recuperar la racionalidad del sistema y lo hacen más compatible con la vida activa».

Lo hemos visto con detalle en el anterior punto — cuadro desarrollado como elaboración propia- por medio de las novedades habidas tanto en el subsidio como entre el endurecimiento de los requisitos para acceder al mismos y su cuantía.

En él se puede ver y traducir que con vistas a garantizar la sostenibilidad del subsidio de "prejubilación» en el largo plazo e incentivar el alargamiento de la vida activa, en el art. 17.7 del RDL 20/2012 se amplía la edad de acceso al mismo a más de 55 años (antes, 52) y se determinan los sistemas para su cómputo (tenerla cumplida «en la fecha del agotamiento de la prestación por desempleo o del subsidio por desempleo» o «en el momento de reunir los requisitos para acceder a un subsidio de los supuestos contemplados en los apartados anteriores o cumplirla durante su percepción») [art. 215.1.3) LGSS]32.

Esta modificación, como regla particular, se aplica a las solicitudes de nacimiento del derecho al subsidio por desempleo que se presenten a partir del 15 de julio de 2012 (disp. fin 13.a.2 RDL 20/2012). Vinculado a ello, también a resultas del RDL 20/2012 (art. 17.8), la extensión máxima en la duración de este subsidio se supedita a que el trabajador alcance la edad «que le permita acceder a la pensión contributiva de jubilación, en cualquiera de sus modalidades» en lugar de la edad "ordinaria exigida en cada caso para causar derecho a la pensión contributiva de jubilación» que regía anteriormente (art. 216.3 LGSS).

Este nuevo régimen opera, igualmente, para las solicitudes de nacimiento del derecho presentadas a partir del 15 de julio de 2012. Por último, el RDL 20/2012 (art. 17.10) da una nueva redacción al art. 218 de la LGSS de la que resulta, por un lado, la sustitución de las referencias a la edad — de 52 a 55 años, recordamos- y, por otro lado, la unificación de la regla de cálculo de la base de cotización que se toma para fijar la cotización a ingresar por la entidad gestora a la Segu- 
ridad Social correspondiente a la contingencia de jubilación (art. 218.3 LGSS): el tope mínimo de cotización vigente en cada momento en cualquier caso.

El precepto se aplica desde el 1 de septiembre de 2012, también para los subsidios preexistentes, que procede recalcular (disp. trans. 5. ${ }^{\mathrm{a}} \mathrm{y}$ disp. fin. $13 .^{\mathrm{a}}$ RDL 20/2012). 4.3.2. Otras medidas.

Junto con las reformas del régimen jurídico del subsidio de "prejubilación», y basándose en los mismos argumentos generales reproducidos en líneas anteriores, la disp. derog. única, apdo. 3.a), del RDL 20/2012 suprime el subsidio especial de mayores de 45 años regulado en el art. 215.1.4) de la LGSS y, consecuentemente, las restantes disposiciones de esta ley referidas al mismo.

Tal medida surte efectos desde el 15 de julio de 2012, aunque se mantiene su aplicación para los desempleados mayores de dicha edad que hubieran agotado la prestación por desempleo de nivel contributivo de 720 días antes de esa fecha (disp. trans. 4. ${ }^{a}$ RDL 20/2012).

Por otro lado, a efectos de determinar el requisito de carencia de rentas del art. 215.1.1) de la LGSS y escudado en que de otro modo «el patrimonio del solicitante sólo se tiene en cuenta de forma indirecta», el RDL 20/2012 (art. 17.7) modifica el art. 215.3.2) de la LGSS mediante la elevación del porcentaje, del 50\% al 100\%, del tipo de interés legal del dinero aplicable al valor de su montante económico.

En esta ocasión, la medida rige para las prestaciones cuyo nacimiento del derecho derive de situaciones legales de desempleo producidas a partir del $15 \mathrm{de}$ julio de 2012.

En tercer lugar, la cuantía del subsidio por desempleo por pérdida de un trabajo a tiempo parcial (art. 217.1 LGSS) pasa a calcularse, según el art. 17.9 del RDL 20/2012, en proporción a las horas previamente trabajadas en lugar de ser, como para el resto de supuestos, igual al $80 \%$ del IPREM mensual vigente en cada momento.

Esta modificación no se aplica ni a emigrantes retornados en los términos del art. 215.1.c) de la LGSS, ni a liberados de prisión conforme al art. 215.1.d) de la LGSS, como tampoco a las solicitudes de nacimiento del derecho al subsidio presentadas antes del 15 de julio de 2012, en virtud de la disp. fin. 13. a 3 del RDL 20/2012.

La única medida que consta en este ámbito, recogida en el art. 17.11 del RD L 20/2012, se incardina en la regulación de las incompatibilidades entre trabajo y prestación o subsidio; concretamente, en relación con el reconocimiento de la compatibilidad con el trabajo a tiempo parcial (art. 221.1 LGSS).

Sobre el particular, se detallan las condiciones aplicables a la deducción en el importe de la prestación o subsidio proporcional al tiempo trabajado. Así, la misma 
procederá, bien cuando el trabajador esté percibiendo la una o el otro como consecuencia de la pérdida de un trabajo a tiempo completo o parcial y obtenga un nuevo contrato a tiempo parcial, bien cuando realice dos contratos a tiempo parcial y pierda uno de ellos, aunque en este segundo caso «la base reguladora de la prestación por desempleo será el promedio de las bases por la que se haya cotizado por dicha contingencia en ambos trabajos durante los 180 días del período a que se refiere el apartado 1 del artículo 210, y las cuantías máxima y mínima a que se refiere el artículo 211 se determinarán teniendo en cuenta el indicador público de rentas de efectos múltiples en función de las horas trabajadas en ambos trabajos».

\section{Propuestas y conclusiones de mejora del subsidio por desempleo}

La actual crisis económica, con cifras de paro superior al $25 \%$, ha provocado que el poder legislativo se plantee la necesidad de analizar la sostenibilidad del Sistema de protección por desempleo para garantizar su continuidad, en el año 2012, que es el año de referencia de la reforma, existía una tasa de paro del $26 \%$, siendo el déficit derivado del pago de prestaciones 13.826 millones de euros (INE, junio de 2012).

Las nuevas modificaciones legislativas muestran una voluntad del legislador por hacer de estas regulaciones un arma para cumplir objetivos presupuestarios, ya que la mayoría de las reformas se han efectuado a través de criterios de gasto.

En la exposición de motivos del RDL 20/2012, de 13 de julio, de medidas para garantizar la estabilidad presupuestaria y de fomento de la competitividad («BOE» de 14 julio) se especifica los objetivos principales de la reforma, siendo ésta la que más incidencia ha tenido en criterios económicos:

- Concentrar la protección en las situaciones de pérdida de empleo y situación personal que requieren especial atención.

- Impulsar la activación de los desempleados incentivando el pronto retorno a la ocupación.

- Generar los incentivos necesarios para asegurar la sostenibilidad del sistema público de prestaciones, contribuir al envejecimiento activo, y facilitar la activación de los trabajadores de más edad.

- Reforzar el sistema de políticas activas sobre la base del principio de eficiencia, permitiendo que los limitados recursos disponibles se destinen a aquellas iniciativas más útiles para desarrollar la empleabilidad de los trabajadores.

- Racionalizar el sistema de prestaciones en su totalidad dotándole de una mayor coherencia interna que asegure su equidad.

En la exposición de motivos continúa evaluando el impacto de la reforma: «La incidencia la han soportado especialmente aquellos gastos más superfluos o 
con efectos más débiles sobre los incentivos de los agentes económicos. En esta línea hay que situar el modelo de prestaciones por desempleo».

En su origen, este subsidio estaba orientado a personas con especiales de dificultades de acceso al mercado laboral, debido principalmente a la edad y a las pocas posibilidades de reinserción laboral. Es un puente hacia la situación de jubilación o prejubilación, puesto que al ser contributivo, hay una exigencia de cotización para el acceso a la jubilación, puesto que a los 52 ańos — ahora con 55 ańos- no hay que olvidar que tienen un carácter contributivo, en tanto en cuanto se exige haber cotizado el tiempo suficiente que permite acceder a la prestación de jubilación.

Según todo lo expuesto anteriormente respecto al subsidio para mayores de 55 ańos existirán tres momentos dependiendo de cuándo se accedió al mismo de conformidad con la actual y vigente normativa:

— Prestación reconocida antes de 15/07/2012. Mayor de 52 años, requisito económico individual y jubilación con la edad ordinaria.

— Prestación reconocida después de 15/07/2012 y antes de 17/03/2013. En resumen: mayor de 55 años, requisito económico individual y jubilación con la edad ordinaria, salvo que tenga derecho a la anticipada.

— Prestación reconocida a partir de 17/03/2013. Mayor de 55 ańos, requisito económico familiar y jubilación con la edad ordinaria, salvo que tenga derecho a la anticipada.

Además se modifica la cotización durante la percepción del subsidio (art. 218 LGSS) desde el 1 de agosto de 2012 la entidad gestora cotizará por la contingencia de jubilación, tanto durante la percepción del subsidio por desempleo para trabajadores mayores de 55 años, como en los casos de perceptores del subsidio por desempleo de trabajadores fijos discontinuos bien se trate de mayores de 55 ańos, bien menores de dicha edad —antes menores de 52 -

La base de cotización de referencia es el tope mínimo de cotización vigente en cada momento y no como hasta ahora en que, en los dos primeros supuestos, se tomaba como base $125 \%$ del ese tope mínimo. Estos trabajadores que tienen fijada como base de cotización el 125 por 100 del tope mínimo pasarán a tener como base el 100 por cien de ese tope mínimo a partir del día 1 de agosto de 2012 (disp. transitoria. 5. ${ }^{\mathrm{a}} \mathrm{RDL}$ )

En conclusiones la actual crisis que nos ocupa y por la que atraviesa España deja un escenario social preocupante, al que se intenta dar respuesta a través de las distintas reformas. Comparado con otros países, el impacto laboral de la crisis en España es especialmente significativo, desde una doble vertiente: la destrucción de empleo y los altas tasas de paro. 
Uno de los objetivos principales de las reformas es la racionalización del sistema de prestaciones, para asegurar la coherencia y equidad eso manifiesta el legislador que la confecciona.

En este sentido, si acudimos a comparar cómo se distribuye el gasto en prestaciones según países, España dedica el 3,1 \% del PIB que se traduce en un 0,15 en conversión gasto/habitante, igual que en Francia, mientras que en Alemania esta cifra es del 0,18\%, y en Dinamarca, del 0,21\%.

Parece que el gasto destinado en España a políticas pasivas está en línea con el resto de países, además de tener en cuenta que según el informe de la OCDE, el salario bruto calculado por paridad, de los países del ejemplo en el año 2011 (inmediatamente anterior a la reforma) son: $26.913 €$, para Espańa; $40.678 €$, para Alemania; $31.110 €$, para Francia, y $42.069 €$, para Dinamarca.

En lo que respecta a la duración de los distintos subsidios y prestaciones, la duración máxima es similar en Espańa a otros países, pues en Alemania puede ser hasta 2 ańos, lo mismo que en Francia, y la reducción de la cuantía por antigüedad en Dinamarca es invariable (hasta $90 \%$ durante todo el periodo), en Holanda del $70 \%$ en el periodo de menor percepción, aunque como dato reseñable en Francia no alcanza el $41 \%$ durante todo el periodo. No parece que comparativamente sea incoherente o insostenible.

Otro de los objetivos clave de las reformas es de dar una respuesta a las personas que necesitan una especial protección. Transcurridos solamente dos años desde la reforma, según datos del INE de junio de 2013 , la reducción en los subsidios supone una disminución de la renta percibida para el $90 \%$ de los beneficiarios.

Según los datos del Servicio Público de Empleo Estatal-SEPE, la tasa de cobertura es de un $65,4 \%$ de los desempleados para el año 2012, frente al $78 \%$ del año 2010 , todo esto con cifras de desempleo constantemente por encima del $25 \%$.

En otro sentido, se introducen en estas reformas otros elementos que generan asimismo desprotección, concretamente la posibilidad de actuación de oficio de la entidad gestora para inspeccionar y/o sancionar a los beneficiarios «cuando se aprecien indicios suficientes de fraude, y la introducción de medidas de control y fiscalización para continuar con la prestación, como es el caso del control de las salidas al extranjero de los beneficiarios, o de dificultar el acceso a la prestación mediante el criterio económico para acceder a la prestación en el que se tiene en cuenta las rentas conjuntas.

Según los datos del Ministerio de Empleo y Seguridad Social, el nivel de cobertura de las prestaciones en los niveles contributivo, no contributivo y asistencial ha sufrido una merma importantísima: Si tenemos en cuenta la secuencia desde el 2008, año en el que comenzó la crisis, hasta el último mes de referencia, vemos que 
la tasa de cobertura para los tres niveles no llega ni al $56 \%$, siguiendo la tasa de desempleo por encima del $24 \%$.

Esto significa que el objetivo de proteger a las personas con especiales necesidades no está cumpliéndose.

Se habla también en las distintas exposiciones de motivos del RDL 20/2012 del propósito de generar los incentivos necesarios para asegurar la sostenibilidad del sistema público de prestaciones, contribuir al envejecimiento activo, y facilitar la activación de los trabajadores de más edad Tras la eliminación del subsidio especial para mayores de 45 años, que tras haber agotado la prestación contributiva, acceden a la asistencial, disminuye la renta de 165.228 perceptores para el año 2012 (el $31 \%$ de los que acceden a la prestación asistencial por agotamiento de la contributiva.

Este colectivo se ve afectado por un lado por el descenso en las prestaciones y por otro por su difícil inserción laboral. Difícil encaje tiene esta realidad con la situación laboral real, puesto que sus posibilidades de encontrar empleo disminuyen con la edad: de los 6,2 millones de parados que había en España en 2012, 1,1 millones son mayores de 50 años, y la cantidad sube hasta los 1,8 millones si se tiene en cuenta el rango entre 45 y 49 años.

En definitiva, las reformas laborales que han ido orientadas a reorganizar el sistema de políticas pasivas no han tenido el resultado esperado. Desde su inicio como hemos visto, han contribuido al empobrecimiento de los perceptores de subsidios y ayudas, recortando su cuantía en unos casos, y dificultando los criterios de acceso y permanencia en otros. Es paradójico que, siendo uno de los principales objetivos la protección de personas que requieren especial atención, se elimine el subsidio para mayores de 45 años, sin que haya correlativamente un desarrollo de una estrategia de empleo para las personas mayores de esta edad que, una vez expulsados del mercado de trabajo, tienen muy difícil recolocación e inserción de nuevo en el mundo laboral.

Tras dos años desde la primera reforma, los niveles de desempleo siguen por encima del $25 \%$, pero la tasa de cobertura por prestación disminuye bien porque se pierden derechos de acceso, bien por eliminar subsidios. Los programas de reinserción no están adecuados al objetivo y apenas cumplen su función, y la desprotección de los trabajadores de mayor edad está en su punto máximo.

Tradicionalmente existe una relación inversamente proporcional entre tiempo de permanencia como beneficiario de prestación y reinserción al mercado laboral, pero estas teorías no pueden funcionar con la situación económico social existente.

Se echan en falta cambios que contribuyan a restaurar el equilibrio de las políticas pasivas, con mejoras para redistribuir los recursos y racionalizarlos con 
una política adecuada a las necesidades reales de la población que quiere trabajar y no puede, y a mejorar el funcionamiento la gestión de las mismas dentro del marco del Estado de Bienestar que impera en Espańa y en su mercado laboral.

No cabe lugar a dudas que este subsidio por desempleo para mayores de 55 ańos es unos de los mas importante para un colectivo de desempleados de difícil entrada de nuevo al sistema del mercado de trabajo. Esta y otras políticas pasivas de empleo deben permanecer en el tiempo y registrar mas y mejor cobertura al sistema de previsión sociolaboral puesto que proviene de un sistema contributivo a un colectivo de difícil reinserción, si ademas tenemos que las políticas activas de empleo y que gestionan los Servicios Publicos de Empleo de las CCAA no son capaces de reactivar a este colectivo expulsado de la vida laboral solo por el hecho de la fecha de nacimiento en su documento nacional de identidad.

\section{Bibliografía consultada}

Blasco Pellicer, A. (2012): "La extinción del contrato de trabajo en el RDL 3/2012: aspectos sustantivos, procesales y de Seguridad Social». En Blasco Pellicer, A.; Camps Ruiz, L.M.; Goerlich Peset, J.M.

Roqueta Buj, R. y Sala Franco, T.: La reforma laboral en el Real Decreto-ley 3/2012. Valencia: Tirant lo Blanch (formato on line).

Blasco Rasero, C. (2012): "La protección social del Estado: ayudas asistenciales a favor de los ciudadanos sin recursos suficientes para vivir», Revista Española de Derecho del Trabajo, 154.

Casas BaAmonde, M.E. y Rodríguez-Piñero y Bravo-Ferrer, M. (2012): Las reformas de la reforma laboral de 2012 (La Ley 3/2012 y el RDL 20/2012), Relaciones Laborales, 15-18 (formato on line).

Fernandez OrRico, F.J. (2012): «Reformas en materia de seguridad y empleo derivadas de la crisis económica (Ley 3/2012), de 6 de julio, y Real Decreto-Ley 20/2012, de 13 de julio", RTSS CEF.

Real Decreto Legislativo 1/1994, de 20 de junio, por el que se aprueba el Texto Refundido de la Ley General de la Seguridad Social.

Real Decreto Legislativo 1/1995, de 24 de marzo, por el que se aprueba el texto refundido de la Ley del Estatuto de los Trabajadores.

LEY 27/2011 sobre actualización, adecuación y modernización del sistema de Seguridad Social.

Real Decreto-ley 20/2012 de 13 de julio, de medidas para garantizar la estabilidad presupuestaria y de fomento de la competitividad (BOE del 14 de julio).

Real Decreto-ley 23/2012, de 24 de agosto, por el que se prorroga el programa de recualificación profesional de las personas que agoten su protección por desempleo. (BOE del 25 de agosto).

Real Decreto-ley 4/2013, de 22 de febrero, de medidas de apoyo al emprendedor y de estímulo del crecimiento y de la creación de empleo (BOE de 23 de febrero).

LeY $11 / 2013$, de 26 de julio, de medidas de apoyo al emprendedor y de estímulo del crecimiento y de la creación de empleo. («BOE» de 27 julio). 